\title{
ON GLOBALLY MINIMAL FOLIATION WITH RESPECT TO LAGRANGIANS ON RIEMANNIAN MANIFOLDS
}

\author{
Tran Viet Dung
}

\section{Introduction}

A foliation of a Riemann manifold is called minimal with respect to a functional if each leaf is minimal with respect to that functional. Volumeminimal foliations of Riemannian manifolds were studied by Harvey, Lawson $[3,4]$, Sullivan [7], Oshikiri [5, 6] and others. We are interested in the minimality of foliations with respect to functionals given by lagrangians. The aim of this paper is to find sufficient conditions for the existence of a lagrangian on a Riemannian manifold such that a given foliation of the manifold is absolutely (or homologically) minimal with respect to the corresponding functional.

\section{§1. Preliminaries}

Let $R^{n}$ be the Euclidean space of dimension $n$. Denote by $\Lambda_{k, n}$ the dual spaces of $k$-vectors and $k$-covectors respectively. The scalar product (,) in $R^{n}$ induces scalar products in $\Lambda_{k, n}$ and $\Lambda^{k, n}$ which are also denoted by (,). Denote the corresponding norm in $\Lambda_{k, n}$ by $|\cdot|$. The comass of the $k$-covector $\omega$ is defined by

$$
\|\omega\|^{*}=\sup \{\omega(\xi) ; \xi \text { is a simple } k \text {-vector, }|\xi|=1\}
$$

and the mass of the $k$-vector $\xi$ is defined by

$$
\|\xi\|=\sup \left\{\omega(\xi) ; \omega \in \Lambda^{k, n},\|\omega\|^{*} \leq 1\right\} .
$$

If $\xi$ is a simple $k$-vector then $\|\xi\|=|\xi|$.

Let $M$ be a Riemannian manifold. Denote by $E^{k} M$ the vector space of all differential $k$-forms on $M$, by $E_{k} M$ the space of all $k$-currents on $M$ with compact support and finite mass. A lagrangian of degree $k$ on $M$ is a continuous mapping $L: \Lambda_{k} M \rightarrow R$ such that its restriction on each fiber $\Lambda_{k} M_{x}$ of the Grassmann bundle $\Lambda_{k} M_{x}$ is positively homogeneous, here $M_{x}$ is the tangent vector space at the point $x \in M$. Each lagrangian of degree $k$ on $M$ defines a functional $J$ on $E_{k} M$ as follows

Received February 5, 2002; revised June 21, 2002. 


$$
J(S)=\int L\left(\vec{S}_{x}\right) d\|S\|(x), \quad S \in E_{k} M,
$$

where $\vec{S}_{x}$ is the tangent $k$-vector of $S$ at $x$ and $\|S\|$ is the variational measure given by $S$ (see [1]). Each oriented compact $k$-surface $V$ on $M$ can be identified with a $k$-current $[V]$ by the formula

$$
V(\varphi)=\int_{V} \varphi, \quad \varphi \in E^{k} M .
$$

In this case the tangent $k$-vector $[\vec{V}]_{x}$ is a simple $k$-vector associated with the tangent space $V_{x}$, i.e. $[\vec{V}]_{x} \wedge v=0$ for every $v \in V_{x}$ and the orientation of $[\vec{V}]_{x}$ is correspondent to the orientation of the tangent space $V_{x}$.

Definition 1.1. Let $J$ be a functional on $E_{k} M$. A current $S \in E_{k} M$ is called absolutely (respectively, homologically) minimal with respect to $J$ if $J(S) \leq J(T)$ for every $T \in E_{k} M$ such that $(S-T)$ is a closed (respectively, exact) current. The following theorem is needed for us.

THEOREM 1.1 (see [2]). Let $\omega$ be an exact (respectively, closed) $k$-form on $M$, $J$ be a functional on $E_{k} M$ given by lagrangian $L$ of degree $k$ on $M$. Let $S$ be a $k$-current in $E_{k} M$. If the following conditions are satisfied

$$
\begin{aligned}
\omega(\xi) & \leq L(\xi) \quad \text { for every } \xi \in \Lambda_{k} M, \\
\omega\left(\vec{S}_{x}\right) & =L\left(\vec{S}_{x}\right),
\end{aligned}
$$

for almost every $x \in M$ in the sense of the measure $\|S\|$, then $S$ is absolutely (respectively, homologically) minimal with respect to $J$.

In this case, sometime we say that $S$ is minimal with respect to the lagrangian $L$.

\section{§2. Globally minimal foliations of a Riemannian manifold}

Let $M$ be a Riemannian manifold of dimension $n$. Denote by $G_{k} M_{x}$ the set of all oriented $k$-dimensional subspaces of $M_{x}$. Put $G_{k} M=\bigcup_{x} G_{k} M_{x}$. Each element of $G_{k} M$ can be identified with a simple $k$-vector of mass one. An oriented $k$-dimensional distribution $P$ of the manifold $M$ is a section $\vec{P}: M \rightarrow$ $G_{k} M$ such that for every $x \in M$ there is a neighbourhood $U$ of $x$ and independent differentiable vector fields $X_{1}, \ldots, X_{k}$ on $U$ being an oriented frame of $\vec{P}$. That is, for each $y \in U$ the basis $\left\{X_{1}(y), \ldots, X_{k}(y)\right\}$ defines the orientation of $\vec{P}(y)$. Let the distribution $P$ be integrable, then each maximal connected oriented integral submanifold is a leaf of a $k$-dimensional oriented foliation $\mathscr{F}$ of $M$.

Definition 2.1. Let $J$ be a functional on $G_{k} M$. The foliation of dimension $k$ on $M$ is said to be absolutely (respectively, homologically) minimal with respect 
to $J$ if a compact domain of each leaf is an absolutely (respectively, homologically) minimal $k$-current with respect to $J$.

THeOREM 2.1. Let $\mathscr{F}$ be a k-dimensional foliation of $M$ given by an oriented distribution $\vec{P}$. If there is an exact (respectively, closed) $k$-form $\theta$ satisfying the condition $\theta(\vec{P}(x)) \neq 0$ for every $x \in M$ then there exists a class of convex differentiable lagrangians of degree $k$ on $M$ such that the foliation $\mathscr{F}$ is absolutely (respectively, homologically) minimal with respect to the corresponding functionals.

Proof. Let $\theta$ be an exact (respectively, closed) $k$-form satisfying $\theta(\vec{P}(x))$ $\neq 0$. From the continuity of $\theta$ and $\vec{P}$ it follows that $\theta(\vec{P}(x))$ has the same sign on each connected component of $M$. Denote by $\omega$ the $k$-form on $M$ defined by the formula

$$
\begin{cases}\omega_{x}=\theta_{x} & \text { if } \theta(\vec{P}(x))>0 \\ \omega_{x}=-\theta_{x} & \text { if } \theta(\vec{P}(x))<0 .\end{cases}
$$

Then the $k$-form $\omega$ is exact (respectively, closed). Moreover,

$$
\omega(\vec{P}(x))>0
$$

for every $x \in M$. Denote by $H$ the hyperplane in $\Lambda_{k} M_{x}$ given by the equation

$$
\omega_{x}(\xi)=\omega_{x}(\vec{P}(x)) .
$$

There is $k$-vector $\omega_{x}^{*}$ in $\Lambda_{k} M_{x}$ satisfying the relation $\left(\omega_{x}^{*}, \xi\right)=\omega_{x}(\xi)$ for every $\xi \in \Lambda_{k} M_{x}$, here $($,$) is the scalar product in \Lambda_{k} M$ induced by the Riemannian metric on $M$. Denote by $d\left(O_{x}, H\right)$ the distance from the zero-vector $O_{x}$ to the hyperplane $H$. We have

$$
d\left(O_{x}, H\right)=\frac{|\omega(\vec{P}(x))|}{\left|\omega_{x}^{*}\right|}
$$

Put

$$
\alpha_{x}=\frac{\omega(\vec{P}(x)) \cdot \omega_{x}^{*}}{\left|\omega_{x}^{*}\right|^{2}} \in \Lambda_{k} M_{x} .
$$

For two arbitrary points $\xi_{1}, \xi_{2}$ in $H$ we have

$$
\begin{aligned}
\left(\alpha_{x}, \xi_{2}-\xi_{1}\right) & =\frac{\omega(\vec{P}(x))}{\left|\omega_{x}^{*}\right|^{2}}\left|\left(\omega_{x}^{*}, \xi_{2}\right)-\left(\omega_{x}, \xi_{1}\right)\right| \\
& =\frac{\omega(\vec{P}(x))}{\left|\omega_{x}^{*}\right|^{2}}\left|\omega_{x}\left(\xi_{2}\right)-\omega_{x}\left(\xi_{1}\right)\right|=0 .
\end{aligned}
$$

Thus, the $k$-vector $\alpha_{x}$ is orthogonal to $H$. It is easy to see that

$$
\left|\alpha_{x}\right|=d\left(O_{x}, H\right) .
$$

Put

$$
\beta_{x}=\vec{P}(x)-\alpha_{x} .
$$


Denote by $C_{L, x}$ the ellipsoid of revolution with focuses $O_{x}, 2 \beta_{x}$ and passing through $\vec{P}(x)$ in $\Lambda_{k} M_{x}$. We can check that $C_{L, x}$ is tangent to $H$ at $\vec{P}(x)$. Consider a mapping $L_{x}: \Lambda_{k} M_{x} \rightarrow R$ positively homogeneous of degree 1 such that for each $\xi \in C_{L, x}$,

$$
L_{x}(\xi)=\omega(\vec{P}(x)) .
$$

Obviously, $L_{x}$ is completely defined by its values on $C_{L, x}$. Put

$$
B_{L, x}=\left\{\xi \in \Lambda_{k} M_{x} ; L_{x}(\xi) \leq \omega(\vec{P}(x))\right\} .
$$

Then the set $B_{L, x}$ is a closed convex domain containing $O_{x}$ in $\Lambda_{k} M_{x}$ and $\partial B_{L, x}=C_{L, x}$. When $x$ moves in the whole $M$ we obtain a mapping $L: \Lambda_{k} M \rightarrow$ $R$ such that

$$
\left.L\right|_{\Lambda_{k} M_{x}}=L_{x} .
$$

Because $\omega(\vec{P}(x))>0$ it follows $L(\xi)>0$ for every $\xi \neq 0$. We will show that $L$ is differentiable. Actually, for each $\xi \neq 0$, in $\Lambda_{k} M_{x}$ there is a number $\lambda>0$ such that $\lambda \xi \in C_{L, x}$, then

$$
L(\xi)=\frac{1}{\lambda} \omega(\vec{P}(x))
$$

The number $\lambda$ is the positive solution of the equation

$$
\left|\lambda \xi-O_{x}\right|+\left|\lambda \xi-2 \beta_{x}\right|=2|\vec{P}(x)| .
$$

It is easy to check that the positive solution $\lambda=\lambda\left(\xi, \omega_{x}, \vec{P}(x)\right)$ of the equation (2.9) is differentiably dependent on $x, \xi$. From (2.8) we have

$$
L(\xi)=\frac{\omega(\vec{P}(x))}{\lambda\left(\xi, \omega_{x}, \vec{P}(x)\right)}
$$

The differentiability of $\vec{P}, \omega, \lambda$ implies the differentiability of $L$. The convexity of $L$ follows the convexity of the set $B_{L, x}$. We will prove that

$$
\omega(\xi) \leq L(\xi)
$$

for every $\xi \in \Lambda_{k} M$.

Let $\xi$ be an arbitrary point of $C_{L, x}$. If there is a number $t>0$ such that $t \xi \in H$ then $t \geq 1$ and

$$
\omega(\xi)=\frac{1}{t} \omega(t \xi)=\frac{1}{t} \omega(\vec{P}(x)) \leq \omega(\vec{P}(x)) .
$$

From (2.7) and (2.12) it follows

$$
\omega(\xi) \leq L(\xi) .
$$

If there is no number $t>0$ such that $t \xi \in H$ then there exists $t<0$ so that $t \xi \in H$, or, the straight line $\left\langle O_{x}, \xi\right\rangle$ is parallel to $H$. In both of these cases, we have $\omega(\xi) \leq 0$. Hence, the innequality $\omega(\xi) \leq L(\xi)$ is satisfied automatically. 
Thus (2.11) is proved for every $\xi \in C_{L, x}$. This implies that (2.11) is true for every $\xi \in \Lambda_{k} M_{x}$. By our construction,

$$
\omega(\vec{P}(x))=L(\vec{P}(x)) .
$$

According to Theorem 1.1, each compact domain of a leaf of $\mathscr{F}$ is absolutely (respectively, homologically) minimal with to the functional given by lagrangian $L$.

In fact, we can find a class of lagrangians $L_{t}, t \geq 0$ analogously. Each lagrangian $L_{t}$ can be defined as above when the set $C_{L, x}$ is exchanged by a set $C_{L, x, t}$ given by the equation

$$
\left|\xi+t \beta_{x}\right|+\left|\xi-2 \beta_{x}-t \beta_{x}\right|=2\left|\vec{P}(x)+t \beta_{x}\right|
$$

The set $C_{L, x, t}$ is the ellipsoid of revolution with focuses $-t \beta_{x}, 2 \beta_{x}+t \beta_{x}$ and passing through $\vec{P}(x)$. Using an argument analogous to the previous one we obtain the lagrangian $L_{t}$ satisfying the conditions

$$
\begin{aligned}
\omega(\xi) & \leq L_{t}(\xi) \\
\omega(\vec{P}(x)) & =L_{t}(\vec{P}(x))
\end{aligned}
$$

Hence, the foliation $\mathscr{F}$ is absolutely (respectively, homologically) minimal with respect to the functional given by $L_{t}$.

Thus, the theorem is proved.

\section{§3. $G$-invariant foliations minimizing lagrangians}

In this section we consider foliations invariant with respect to the action of a connected compact Lie group. We say that the Lie group $G$ acts on the Riemannian manifold if there is a mapping of class $C^{\infty}$

$$
\Pi: G \times M \rightarrow M
$$

such that for every $g \in G$ the mapping $\Pi_{g}: M \rightarrow M$ defined by

$$
\Pi_{g}(x)=\Pi(g, x)
$$

is a diffeomorphism from $M$ into itself. Moreover, $\forall g, h \in G, \Pi_{g \cdot h}=\Pi_{g} \cdot \Pi_{h}$ and $\Pi_{e}=\mathrm{id}_{M}$ where $e$ is the unity of $G$. As usual, the notation $g x$ is used instead of $\Pi(g, x)$. Each $g \in G$ induces a mapping

$$
g_{*}: G_{k} M \rightarrow G_{k} M .
$$

Definition 3.1. Assume that the Lie group $G$ acts transitively on $M$. Let $\vec{P}$ be a $k$-dimensional distribution on $M$. The distribution $\vec{P}$ is said to be invariant with respect to the action of $G$ if for every $x \in M, g \in G$, the equality

$$
\vec{P}(g x)=g_{*} \vec{P}(x)
$$

holds. 
In this case the foliation $\mathscr{F}$ corresponding to $\vec{P}$ is said to be invariant with respect to the action of $G$ (or, $G$-invariant). Each $\Pi_{g}, g \in G$, induces a mapping

$$
g^{*}: E^{k} M \rightarrow E^{k} M
$$

and a mapping

$$
g_{*}: E_{k} M \rightarrow E_{k} M .
$$

Definition 3.2. A differential $k$-form $\omega$ (respectively, $k$-current $S$ ) is said to be invariant with respect to the action of $G$ if $g^{*} \omega=\omega$ (respectively, $g_{*} S=S$ ) for every $g \in G$.

Let $L$ be a lagrangian of degree $k$ on $M$. The lagrangian $L$ is said to be $G$-invariant if $L\left(g_{*} \xi\right)=L(\xi)$ for every $g \in G$.

In [1] Dao Trong Thi has shown that for a fixed current $S \in E_{k} M$, the mapping $\rho: G \rightarrow E_{m} M$ given by $\rho(g)=g_{*} S$ is continuous. For the compact Lie group $G$, there exists a bilaterally invariant Haar's measure on $G$ such that the measure of the whole $G$ equals to 1 . For a $k$-form $\omega \in E^{k} M$, we put

$$
\Pi_{G}^{*} \omega=\int_{G} g^{*} \omega d g
$$

and for $k$-current $S \in E_{k} M$, put

$$
\Pi_{G} \omega=\int_{G} g_{*} S d g
$$

It is easy to see that $\Pi_{G}^{*} \omega$ (respectively, $\left.\Pi_{G} \omega\right)$ is $G$-invariant. Note that the action $g_{*}$ (respectively, $g^{*}$ ) is comutative with the operator $\partial$ (respectively, $d$ ). Hence if the $k$-form $\omega$ is exact (respectively, closed) then $\Pi_{G}^{*} \omega$ is also exact (respectively, closed).

THEOREM 3.1. Let $G$ be a connected compact Lie group which acts transitively on $M, \mathscr{F}$ be a k-dimensional foliation of $M$ given by an oriented $G$-invariant distribution $\vec{P}$. If there exists an exact (respectively, closed) differential $k$-form $\omega$ on $M$ such that $\omega\left(\vec{P}\left(x_{0}\right)\right) \neq 0$ for some point $x_{0} \in M$ and the sign of $\omega(\vec{P}(x))$ does not exchange in $M$ then there is a class of $G$-invariant differentiable convex lagrangians of degree $k$ on $M$ such that $\mathscr{F}$ is absolutely (respectively, homologically), minimal with respect to the corresponding functionals.

Proof. Consider the $k$-form $\tilde{\omega}=\Pi_{G}^{*} \omega$ on $M$. It is an exact (respectively, closed) $k$-form. We have

$$
\begin{aligned}
\tilde{\omega}(\vec{P}(x)) & =\int_{G} g^{*} \omega(\vec{P}(x)) d g=\int_{G} \omega\left(g_{*} \vec{P}(x)\right) d g \\
& =\int_{G} \omega(\vec{P}(x)) d g .
\end{aligned}
$$


Because $\omega\left(\vec{P}\left(x_{0}\right)\right) \neq 0$ and the sign of $\omega(\vec{P}(x))$ does not exchange in $M$, $\tilde{\omega}(P(x)) \neq 0$ for every $x \in M$.

Analogously as in the proof of Theorem 2.1, we can construct a differentiable, convex, positively homogeneous function $L_{t, x_{0}}$ on $\Lambda_{k} M_{x_{0}}$ for each parameter $t \geq 0$ such that the following conditions are satisfied

$$
\tilde{\omega}(\xi) \leq L_{t, x_{0}}(\xi)
$$

for every $\xi \in \Lambda_{k} M_{x_{0}}$, and

$$
\tilde{\omega}\left(\vec{P}\left(x_{0}\right)\right)=L_{t, x_{0}}\left(\vec{P}\left(x_{0}\right)\right)
$$

Denote by $H_{x_{0}}$ the subgroup of $G$ consisting of elements that withstand the point $x_{0}$. On $H_{x_{0}}$ there exists the Haar's bilaterally invariant measure such that the measure of $H_{x_{0}}$ equals 1 . Consider the mapping $\tilde{L}_{t, x_{0}}: \Lambda_{k} M_{x_{0}} \rightarrow R$ given by the formula

$$
\tilde{L}_{t, x_{0}}(\xi)=\int_{H_{x_{0}}} L_{t, x_{0}}\left(h_{*} \xi\right) d h
$$

where $\xi \in \Lambda_{k} M_{x_{0}}$. Then $\tilde{L}_{t, x_{0}}$ is $H_{x_{0}}$-invariant. Since $\tilde{\omega}$ is $G$-invariant, $\tilde{\omega}_{x_{0}}$ is $H_{x_{0}}$-invariant. From (3.5), it follows

$$
\tilde{\omega}_{x_{0}}\left(h_{*} \xi\right) \leq L_{t, x_{0}}\left(h_{*} \xi\right)
$$

for every $\xi \in \Lambda_{k} M_{x_{0}}, h \in H_{x_{0}}$. Hence,

$$
\tilde{\omega}_{x_{0}}(\xi)=\int_{H_{x_{0}}} \omega\left(h_{*} \xi\right) d h \leq \int_{H_{x_{0}}} L_{t, x_{0}}\left(h_{*} \xi\right) d h=\tilde{L}_{t, x_{0}}(\xi) .
$$

Analogously,

$$
\tilde{\omega}_{x_{0}}\left(\vec{P}\left(x_{0}\right)\right)=\tilde{L}_{t, x_{0}}\left(\vec{P}\left(x_{0}\right)\right) .
$$

The convexity of $L_{t, x_{0}}$ implies the convexity of $\tilde{L}_{t, x_{0}}$. Denote by $\tilde{L}_{t}$ a mapping $\tilde{L}_{t}: \Lambda_{k} M \rightarrow R$ given by

$$
\tilde{L}_{t}\left(g_{*} \xi\right)=\tilde{L}_{t, x_{0}}(\xi)
$$

for every $g \in G, \xi \in \Lambda_{k} M_{x_{0}}$. The mapping $\tilde{L}_{t}$ is defined correctly. Actually, if $g_{*} \xi=g_{*}^{\prime} \cdot \xi^{\prime}$ then $\xi^{\prime}=\left(g^{\prime-1} g\right)_{*} \xi$. It is easy to see that $\left(g^{\prime-1} g\right) \in H_{x_{0}}$. Since $\tilde{L}_{t, x_{0}}$ is $H_{x_{0}}$-invariant, $\tilde{L}_{t, x_{0}}\left(\xi^{\prime}\right)=\tilde{L}_{t, x_{0}}(\xi)$. Thus, $\tilde{L}_{t}$ is dependent on only the product $g \xi$. Then $\tilde{L}_{t}$ is a $G$-invariant, convex, differentiable lagrangian. From (3.9), (3.10) and the $G$-invariance of $\tilde{\omega}, \tilde{L}_{t}, \vec{V}$, it follows

$$
\tilde{\omega}(\xi) \leq L_{t}(\xi)
$$

for every $\xi \in \Lambda_{k} M$ and

$$
\tilde{\omega}(\vec{P}(x))=\tilde{L}_{t}(\vec{P}(x))
$$

According to Theorem 1.1, the foliation is absolutely (respectively, homologically) minimal with respect to the functional corresponding to $L_{t}$, for each $t \geq 0$.

This completes the proof of theorem. 


\section{REFERENCES}

[1] Dao Trong Thi, Globally minimal currents and surfaces in Riemannian manifolds, Acta Math. Vietnam., 10 (1985), 296-333.

[2] Dao Trong Thi, Minimal real currents on compact Riemannian manifolds, Izv. Acad. Nauk SSSR Ser. Mat., 41 (1977), 807-820.

[ 3 ] R. Harvey and H. B. Lawson, JR., Calibrated foliations, J. Math. Amer., 103 (1981), 411435.

[4] R. Harvey and H. B. Lawson, JR., Calibrated geometries, Acta Math., 148 (1982), 47-157.

[ 5] G. Oshikiri, A remark on minimal foliations, Tôhoku Math. J. (2), 33 (1981), 133-137.

[6] G. Oshikiri, Some remarks on minimal foliations, Tôhoku Math. J. (2), 39 (1987), 223-229.

[7] D. Sullivan, A homological characterization of foliations consisting of minimal surfaces, Comment. Math. Helv., 54 (1979), 218-223.

Departement of Mathematics

Hanoi University of Technology, Vietnam

Khoa Toan, Dai hoc Bach khoa Hanoi, Vietnam

e-mail: tvdung_g@yahoo.com 